\title{
Electromagnetomotive force fields in noninertial reference frames and accelerated superconducting quantum interferometers
}

\author{
Uwe R. Fischer, Christoph Häussler, Jörg Oppenländer, and Nils Schopohl \\ Eberhard-Karls-Universität Tübingen, Institut für Theoretische Physik \\ Auf der Morgenstelle 14, D-72076 Tübingen, Germany
}

(Dated: November 10, 2018)

\begin{abstract}
We discuss the prospects of detecting with high precision the force fields related to noninertiality in superconducting circuits. Special emphasis is laid on the perfectly conducting and perfect diamagnetism analogues of the Tolman-Stewart respectively Barnett effects. The influence of acceleration and rotation on the electrodynamics of superconducting interferometers is explicitly described. In particular, we show how motion induced changes of the oscillation frequency of the local Josephson oscillators in superconducting quantum interference filters can be used for precision measurements of acceleration in free space.
\end{abstract}

\section{INTRODUCTION}

The detection of electromagnetic fields induced by acceleration in magnetizable materials and metals has a history reaching back more than one century $\stackrel{\underline{1}}{\underline{1}}$. Two famous experiments stand out in this respect. In 1915, Barnett measured the magnetic field of a magnetizable material induced by rotating it ${ }^{2}$. One year later, Tolman and Stewart measured the electromotive force if metals are (linearly) accelerated ${ }^{3}$. They thereafter concluded on the effective mass of the current carrier ${ }^{4}$, which turned out be somewhat different from that of the electron in vacuo. A more modern experiment with increased precision in rotationally accelerated conductors has been carried out ${ }^{5}$, where it was found that the mass is the electron mass in vacuum to within about one percent $t^{6}$.

Superconductors and, more generally, macroscopically coherent quantum systems, are distinguished by the fact that the mass of the current carriers has been measured, within currently achievable precision of a few ppm to be exactly twice the bare mass $m_{e}$ of the electrons in vacuo. The mass is subject to very small (relativistic) corrections only, so small as to currently elude precise experimental determination. The measurement of the bare mass proceeds via the magnetomechanical effect in superconductors, the London moment ${ }^{7.8 .9}$. The London magnetic field induced by rotation (cf. equation (13) below) is proportional to the rotation velocity and to the ratio of twice the mass $m$ of the superconducting current carriers divided by their charge $q$. The best measurement to date $\frac{10}{}$ yielded for the current carrying Cooper pairs a mass $m / 2 m_{e}=1.000084(21)$.

The London moment is a universal magnetomechanical property of rotating superconductors, independent of specific material properties, and verified not only in conventional superconductors, but also in the high- $T_{C} \frac{11}{c}$ and heavy fermion species $\frac{12}{2}$. It furnishes a generalization of the familiar phenomenon of Meissner screening to noninertial, material reference frames of the superconducting state. The London moment, then, represents a par- ticularly striking instance of a quantum protectorate 13 , for which the phenomenon of macroscopic quantum coherence (the fact that the quantum of action $h=2 \pi \hbar$ appears within a macroscopically measurable quantity) "protects" the bare property of a particle. It is this phenomenon which enables the precise measurements of bare quantities related to the charge and mass of the electron. In the Quantum Hall effect, one measures in effect the fine structure constant $e^{2} / 4 \pi \epsilon_{0} \hbar c$, which can be determined to an accuracy of $0.1 \mathrm{ppb}$ in comparative measurements between two Hall probes 14 . In superconducting quantum interference devices (SQUID), the quantum of (Cooper pair) flux $\Phi_{0}=h / 2|e|$ is used as a standard to measure magnetic fields with unprecedented precision. The properties of Josephson junctions also made possible, e.g., the confirmation of constancy of the electrogravitochemical potential (as opposed to the conventional electrochemical potential without the inclusion of a gravitational contribution), in a circuit with two Josephson junctions separated $7.2 \mathrm{~cm}$ in height, which amounts to perpetuating a voltage constancy of $10^{-22}$ Volts over a time span of ten hours 15 . Correspondingly, due to the fact that the ratio $m / 2|e|$ is on a level of at least one ppm the bare ratio of the vacuum, a superconductor should be able to measure its own state of rotation and, more general, acceleration with very high accuracy.

In the following, we give an account of the influence of noninertial forces on macroscopic quantum devices, as specifically represented by SQUIDs and Josephson junction arrays. This includes a study of the perfectly conducting and perfect diamagnetism analogues of the Tolman-Stewart respectively Barnett effects, the latter effect in the superconductor being represented by the London moment. The prospects of detecting with high precision the force fields related to noninertiality are given.

The fact that the exact bare mass appears in the London equation, which relates mechanical and magnetic quantities, implies that an effective theory, describing the motion of the massive current carriers, may be construed 
in a particularly transparent way. Specifically, in the linear in velocity, nonrelativistic limit and for small deviations from the Minkowski metric of flat space-time, a general gauge invariance principle can be satisfied ${ }^{16}$, which puts mechanical and proper electromagnetic forces on an equal footing, uniting them into electromagnetomotive forces. This program of generalized gauge invariance is described in the following section III on the basis of nonrelativistic kinematics. In an appendix, we outline the derivation of this gauge invariance program extracted from relativistic geodesic motion, and relate the potentials of the noninertial force fields to metric coefficients in weakly perturbed Minkowski space-time. In section ஹII we then describe the influence of electromagnetomotive force fields on the electrodynamics of superconducting quantum interferometers. In particular, the influence of acceleration and rotation on the voltage response function of one-dimensional Josephson junction arrays is discussed. For special Josephson junction arrays, so-called superconducting quantum interference filters ${ }^{17.18 .19}$, it is explicitly shown how such devices can be used for precision measurements of rotation. The knowledge of the electromagnetomotive force fields in the superconductor enables as an application the sensitive tracking of the trajectory of the quantum interference device. The procedure to be used for that purpose will be outlined in section IV]

\section{ELECTROMAGNETOMOTIVE FORCE FIELDS FOR ACCELERATED SAMPLES IN RIGID BODY ROTATION}

The noninertial force on a massive test particle inside a rotating and accelerating probe, as measured in the probe's rest frame, is given by the standard expression

$$
\begin{aligned}
\boldsymbol{F}_{\text {noninertial }}= & 2 m \boldsymbol{v} \times \boldsymbol{\Omega}-m \boldsymbol{\Omega} \times \boldsymbol{\Omega} \times \boldsymbol{r}-m\left(\partial_{t} \boldsymbol{\Omega}\right) \times \boldsymbol{r} \\
& -m \nabla \Phi-m \partial_{t}^{2} \boldsymbol{r}_{0}
\end{aligned}
$$

where $\boldsymbol{\Omega}$ is the rotation velocity; $-m \nabla \Phi$ is a possible scalar force on the particle, e.g., gravity, and $\Phi$ its potential. The first term on the right hand side of (11) represents (minus) the Coriolis force, the second one the centripetal force, and the third term is due to temporal changes of the angular velocity. The vector $\boldsymbol{r}_{0}$ is the position of the center of rotation, and $\partial_{t}^{2} \boldsymbol{r}_{0}$ is an (externally imposed) linear acceleration of this center of rotation (cf. Fig. (1).

Compare the relation (11) to the expression for the Lorentz force:

$$
\boldsymbol{F}_{\text {Lorentz }}=q \boldsymbol{v} \times \boldsymbol{B}+q \boldsymbol{E}, \quad(\text { Lorentz) }
$$

where as usual, provided that the conventional homogeneous Maxwell equations

$$
\operatorname{rot} \boldsymbol{E}=-\partial_{t} \boldsymbol{B}, \quad \operatorname{div} \boldsymbol{B}=0
$$

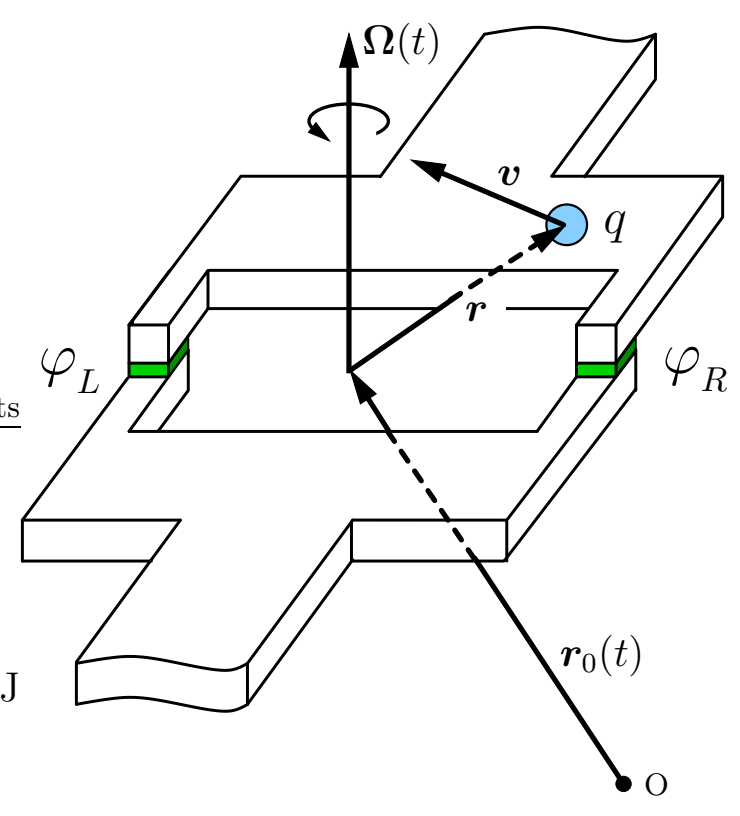

FIG. 1: A Cooper pair in a rotating and accelerating superconducting quantum interference device (SQUID), with two Josephson junctions. Its position and velocity are given by their values $\boldsymbol{r}$ and $\boldsymbol{v}$ in the frame rotating with angular velocity $\boldsymbol{\Omega}$ about a prescribed axis, which is located at a (time dependent) laboratory frame position $\boldsymbol{r}_{0}$. For representation purposes, the axis is in this picture located at the center of the SQUID and perpendicular to its surface. $\varphi_{L}$ and $\varphi_{R}$ are the gauge invariant phase differences associated to the Josephson junctions.

hold, the magnetic and electric fields are derivable from vector and scalar potentials as

$$
\boldsymbol{E}=\nabla A_{0}-\partial_{t} \boldsymbol{A}, \quad \boldsymbol{B}=\nabla \times \boldsymbol{A} .
$$

We, then, define vector and scalar potentials associated

to noninertiality as follows

$$
\boldsymbol{a}=\boldsymbol{\Omega} \times \boldsymbol{r}+\partial_{t} \boldsymbol{r}_{0}, \quad a_{0}=\frac{1}{2} \Omega^{2} \boldsymbol{r}_{\perp}^{2}-\Phi,
$$

where $\boldsymbol{r}_{\perp}$ is the distance vector perpendicular to the axis of rotation. Summing the mechanical and electromagnetical forces, we may infer that for a charged massive particle like electron $(q=-|e|)$ or Cooper pair $(q=-2|e|)$, we can merge the above potentials and the electromagnetic potentials into a generalized vector potential, incorporating the coupling constants charge $q$ and mass $m^{20}$,

$$
\mathcal{A}=q \boldsymbol{A}+m \boldsymbol{a},
$$

and a generalized scalar potential

$$
\chi=-q A_{0}-m a_{0} .
$$


The sum of the generalized electromotive and magnetomotive forces, acting on an electron, consisting of noninertial plus proper Lorentz and electric forces, then takes on the form

$$
\boldsymbol{F}_{\mathcal{L}}=\mathcal{E}+\boldsymbol{v} \times \mathcal{B}
$$

where the generalized electric and magnetic fields are given by the potentials $\mathcal{A}$ and $\chi$ :

$$
\begin{aligned}
\mathcal{E} & =-\nabla \chi-\partial_{t} \mathcal{A} \\
& =q \boldsymbol{E}+m \nabla a_{0}-m \partial_{t} \boldsymbol{a} \\
& =q \boldsymbol{E}-m \boldsymbol{\Omega} \times(\boldsymbol{\Omega} \times \boldsymbol{r})-m\left(\partial_{t} \boldsymbol{\Omega}\right) \times \boldsymbol{r}-m \nabla \Phi-m \partial_{t}^{2} \boldsymbol{r}_{0}, \\
\mathcal{B} & =\nabla \times \mathcal{A} \\
& =q \boldsymbol{B}+2 m \boldsymbol{\Omega} .
\end{aligned}
$$

As a consequence of relation (8) for the total force, the usual expression for the drift velocity of the charge carriers, resulting from zero total force in perpendicular electric and magnetic fields, experiences the obvious modification that $\boldsymbol{E} \rightarrow \mathcal{E}$ and $\boldsymbol{B} \rightarrow \mathcal{B}$, so that $\boldsymbol{v}_{D}=\mathcal{E} \times \mathcal{B} / \mathcal{B}^{2}$.

The generalized electromagnetic force fields displayed in equations (8) and (9) give a theory possessing in effect two $\mathrm{U}(1)$ gauge symmetries. The standard $\mathrm{U}(1)$ from electromagnetism, with coupling constant $q$ (charge), and another U(1) gauge symmetry, with coupling constant $m$ (inertial rest mass). The gauge potential of this second $\mathrm{U}(1)$ has a scalar part $a_{0}$ and a vectorial part $a_{i}$. The homogeneous Maxwell equations

$$
\begin{aligned}
\operatorname{rot} \mathcal{E} & =-\partial_{t} \mathcal{B} \\
\operatorname{div} \mathcal{B} & =0
\end{aligned}
$$

then follow from the existence of the generalized potentials $\mathcal{A}$ and $\chi$ in (6) and (7), which give the fields $\mathcal{E}$ and $\mathcal{B}$ in (9). They are identical to the conventional homogeneous Maxwell equations in (3) with the replacements $\boldsymbol{E} \rightarrow \mathcal{E}$ and $\boldsymbol{B} \rightarrow \mathcal{B}$. That the Faraday law (10) holds is due to our admitting a variation of the angular velocity with time and the resulting force term in (11). We stress that the fields $\mathcal{E}$ and $\mathcal{B}$ are both referring to the frame co-rotating as well as co-moving with the quantum interference device with respect to the laboratory frame. The laboratory frame velocity $\partial_{t} \boldsymbol{r}_{0}$ as well as the rotation rate $\boldsymbol{\Omega}$ can be time dependent in an arbitrary manner.

The gauge invariant particle (mass) current induced by the electromotive force field is in linear response

$$
\boldsymbol{J}_{i}^{\text {ind }}=\tilde{\boldsymbol{\sigma}}_{i j} \mathcal{E}_{j} .
$$

Observe that the left hand side contains the induced mass current density rather than the electric current density. The associated response coefficient $\tilde{\boldsymbol{\sigma}}_{\boldsymbol{i}}$ is measured in units of $[\tilde{\sigma}]=\left[\sigma_{e l} / q^{2}\right]$. In the case of two coupling constants, $m$ and $q$, it is the number of particles crossing a unit area per unit time, which is the relevant observable. This quantity is proportional to the electromotive force field $\mathcal{E}$, which causes these particles to move.
Evidence for the necessity of using a particle transport equation in the form of (12) comes from the existence of the London field in superconductors. Complete expulsion of the field $\mathcal{B}$ deep inside in a superconductor requires the particle conductivity $\tilde{\boldsymbol{\sigma}}$ to have a contribution proportional to $1 / i \omega$, which yields a term on the right hand side of (12), proportional to the generalized vector potential $\mathcal{A}$. Corresponding to complete Meissner type screening, $\mathcal{B}=\operatorname{rot} \mathcal{A}=q \boldsymbol{B}+2 m \boldsymbol{\Omega}=0$, the London spontaneous field $\boldsymbol{B}_{L}$ then takes the value

$$
\boldsymbol{B}_{L}=-2 \frac{m}{q} \boldsymbol{\Omega} .
$$

This relation corresponds to zero winding number of the (9) phase $\theta$, cf. equations (15)-(18) below. Equation (13) was derived by F. London ${ }^{7}$, and has been verified experimentally already 35 years $\operatorname{ago}^{-}{ }^{-}$, in an experiment in which it was used to infer the Compton wavelength of superconducting electrons. If we insert on the left hand side of the equation (13) the bare electron values $m=2 m_{e}$ and $q=-2|e|$, we have

$$
\left|\boldsymbol{B}_{L}\right|=7.15 \cdot 10^{-11} \text { Tesla }
$$

for $|\boldsymbol{\Omega}|=2 \pi /$ sec. Quantum coherence properties are expressed by the requirement for the line integral of collective particle momentum along a closed path to be quantized:

$$
\oint_{\mathcal{C}}\langle\boldsymbol{p}, d \boldsymbol{r}\rangle=N_{v} h
$$

where $N_{v}$ is the winding number of phase $\theta$, so that the total canonical momentum

$$
\begin{aligned}
\boldsymbol{p} & \equiv \hbar \nabla \theta \\
& =m \boldsymbol{v}_{s}+\mathcal{A} \\
& =m \boldsymbol{v}_{s}+m \partial_{t} \boldsymbol{r}_{0}+m \boldsymbol{\Omega} \times \boldsymbol{r}+q \boldsymbol{A},
\end{aligned}
$$

where $\boldsymbol{v}_{s}$ is the Cooper pair velocity field. It has a mechanical contribution proportional to $m$ and a proper electromagnetic contribution $q \boldsymbol{A}$. The uniqueness condition of the collective phase represented in (15) then leads to the quantization of the sum of a Sagnac flux ${ }^{21.22}$ and the magnetic flux

$$
\begin{aligned}
\Phi & =\oint_{\mathcal{C}}\langle\mathcal{A}, d \boldsymbol{r}\rangle \\
& =q \oint_{\mathcal{C}}\langle\boldsymbol{A}, d \boldsymbol{r}\rangle+m \oint_{\mathcal{C}}\langle\boldsymbol{\Omega} \times \boldsymbol{r}, d \boldsymbol{r}\rangle \\
& =q \int\langle\boldsymbol{B}, d \boldsymbol{S}\rangle+2 m \int\langle\boldsymbol{\Omega}, d \boldsymbol{S}\rangle \\
& =\int\langle\mathcal{B}, d \boldsymbol{S}\rangle=N_{v} h,
\end{aligned}
$$

if we take a path in the bulk of the electron liquid, for which the integral of $m \boldsymbol{v}_{s}$ may be neglected. This flux quantization rule associated with the field $\mathcal{B}$ corresponds 
to the fact that a vortex, represented by a zero in the (collective) electron wave function, where the phase $\theta$ becomes singular, is fundamentally characterized by its winding number $N_{v}$ alone. No properties of the medium in which it lives, in particular the mass and charge of the medium's constituents, enter the quantum of generalized flux, which is given by Planck's quantum of action alone. The relation for the London moment in (13), expressing vanishing magnetomotive force field, corresponds to zero winding number of the phase $\theta$. The classical property of zero generalized magnetic field $\mathcal{B}=0$ expressed by the London moment is hence rooted in the generalized Meissner prescription $N_{v}=0$, and thus relates to the quantum coherence property expressed by (18).

The vanishing of the field $\mathcal{E}$ in the bulk of a noninertial superconductor (in the static limit of zero frequency) implies that the proper electric field is nonzero inside the superconductor, and given by

$$
\begin{aligned}
\boldsymbol{E}_{T} & =\frac{m}{q}\left[-\nabla a_{0}+\partial_{t} \boldsymbol{a}\right] \\
& =\frac{m}{q}\left(\boldsymbol{\Omega} \times \boldsymbol{\Omega} \times \boldsymbol{r}+\partial_{t} \boldsymbol{\Omega} \times \boldsymbol{r}+\partial_{t}^{2} \boldsymbol{r}_{0}+\nabla \Phi\right) \equiv-\frac{m}{q} \boldsymbol{g} .
\end{aligned}
$$

It is composed of the centrifugal, time variation of $\Omega, \Phi$ potential and linear acceleration parts. If the total acceleration $\boldsymbol{g}$ is the gravitational acceleration on the surface of the earth, $|\mathbf{g}|=9.81 \mathrm{~m} / \mathrm{sec}^{2}$, we have the value

$$
\left|\boldsymbol{E}_{T}\right|=5.58 \cdot 10^{-11} \mathrm{Volt} / \mathrm{m}
$$

for the electric field induced in the superconductor. The total electromotive force field is thus simply

$$
\begin{aligned}
\mathcal{E} & =q\left(\boldsymbol{E}-\boldsymbol{E}_{T}\right) \\
& =q \boldsymbol{E}+m \boldsymbol{g},
\end{aligned}
$$

and takes a form analogous to the total magnetomotive force field

$$
\begin{aligned}
\mathcal{B} & =q\left(\boldsymbol{B}-\boldsymbol{B}_{L}\right) \\
& =q \boldsymbol{B}+2 m \boldsymbol{\Omega} .
\end{aligned}
$$

The fact that there is an electric field associated to acceleration (which may be material dependent for nonperfect conductors) was measured by Tolman and Stewart in metals ${ }^{3}$. The general phenomenon associated to $\mathcal{B}$, i.e., the occurrence of a magnetic field if the sample is rotated, was observed for magnetizable materials by Barnett $^{2}$, with a (possibly anisotropic) ratio of magnetic field and rotation different from the one displayed by superconductors expressible via the London equation (13). The Tolman field $\boldsymbol{E}_{T}$ is a property of (in the limit of zero frequency) perfect conductors. The London field $\boldsymbol{B}_{L}$, in turn, is a signature of perfect diamagnetism for rotating samples. Perfect diamagnetism may thus be understood as a hallmark of superconductors in general, be they considered in an inertial or noninertial reference frame. In short: The field $\boldsymbol{E}_{T}$ is measured if $\mathcal{E}$ is vanishing and $\boldsymbol{B}_{L}$ is detected if $\mathcal{B}$ is completely (Meissner) screened.

\section{ACCELERATED JOSEPHSON JUNCTION ARRAYS}

Devices based on superconductive quantum interference can be used as ultrasensitive detectors for magnetic fields. They consist of one, two or even a plurality of Josephson junctions or weak links which are connected as an array to form one or several superconducting loops. Prominent exponents are devices containing two junctions per loop, like the dc-SQUID shown in Fig. 1] It consists of two junctions shunted parallel to form one single loop. Other devices of this class are series arrays of dcSQUIDs or one-dimensional (1D) parallel arrays which can contain a plurality of Josephson junctions. A superconducting interferometer based on an 1D parallel array is shown schematically in Fig. 2

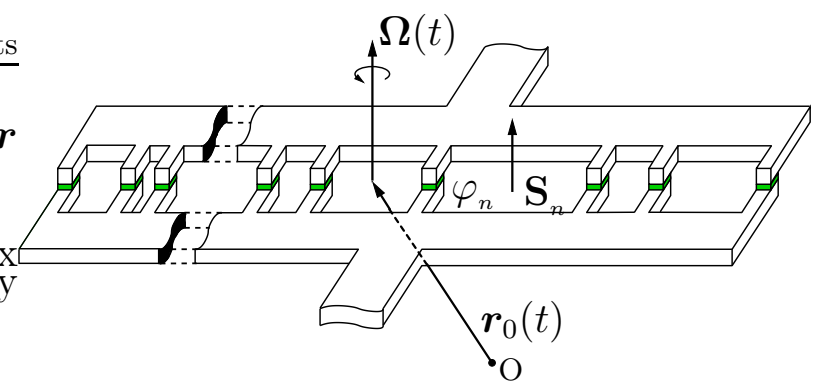

FIG. 2: Superconducting quantum interferometer with $N$ Josephson junctions. Its local frame rotates with angular velocity $\boldsymbol{\Omega}$ about a prescribed axis, which is located at a (time dependent) laboratory frame position $\boldsymbol{r}_{0}$. For representation purposes, the axis is in this picture located at the center of the interferometer and perpendicular its plane surface. $\varphi_{n}$ is the gauge invariant phase difference associated to the $n$th Josephson junction in the array and $\mathbf{S}_{n}$ denotes the $n$th orientated area element.

It consists of $N$ Josephson junctions shunted parallel in such a way that there are $N-1$ individual superconducting loops. In general the $N-1$ areas $S_{n}$ of these superconducting loops can have different shapes and sizes. In particular, judiciously choosing the distribution of the area loop sizes in a suitable unconventional way, 1D parallel arrays can be used as sensors of absolute strength and orientation of magnetic fields. This is due to the fact that 1D parallel arrays are magnetic field to voltage converters, if they are driven by a bias current of suitable strength. Because of their unique response to applied magnetic fields, such 1D parallel arrays with unconventional grating are named quantum interference filters (SQIF) and are explained in greater detail in 17,18 .

In the following the Josephson junctions are assumed to be short junctions such that any spatial variations of the current density along the barriers of the weak links can be safely neglected. In this case each junction can 
be described by a gauge invariant phase difference

$$
\varphi=\theta_{1}-\theta_{2}+1 / \hbar \int_{1}^{2}\langle\mathcal{A}, d \mathbf{r}\rangle
$$

of the macroscopic BCS pairing wave functions on either side of the weak link labeled 1 and 2 respectively. Within the range of validity of the resistively and capacitively shunted junction (RCSJ) model23 the current through the Josephson junction $I$ is a superposition of the dissipationless macroscopic supercurrent $I_{s}$ with a normal current, characterized by a shunt resistance $R$ and shunt capacitance $C$

$$
I(\varphi)=\frac{\hbar C}{2|e|} \partial_{t}^{2} \varphi+\frac{\hbar}{2|e| R} \partial_{t} \varphi+I_{c} \sin (\varphi) .
$$

For an ideal $S-I-S$ junction the supercurrent is connected to the phase difference $\varphi$ across the tunneling barrier by $I_{s}=I_{c} \sin (\varphi)$, where $I_{c}$ is the maximum dissipationless supercurrent, that can flow through the junction. Of course, in 1D arrays each junction can have individual parameters $R_{n}, C_{n}$ and $I_{c, n}$.

In (23) there appears the generalized vector potential $\mathcal{A}$ from (6) in the definition of the gauge invariant phase difference. According to (9), this indicates that, in principle, the electro- and magnetomotive force fields $\mathcal{E}$ and $\mathcal{B}$ can be measured by superconducting quantum interferometers. For 1D parallel Josephson junction arrays, the basic relations are now discussed.

According to the fundamental Josephson relation the rate of change of the time dependent phase difference $\varphi(t)$ is related to the electromotive force field across the junction barrier by

$$
\hbar \partial_{t} \varphi(t)=-\int_{1}^{2}\langle\mathcal{E}, d \mathbf{r}\rangle .
$$

In the case of a electromotive force field $\mathcal{E}$ the Josephson frequency $\nu$ for a single junction evaluates from the right hand side integral of (25), which is the work functional associated with the electromotive force field:

$$
h \nu=\hbar \lim _{t \rightarrow \infty} \frac{1}{t}[\varphi(t)-\varphi(0)] .
$$

Scaling this in experimentally relevant units, we have that the electric field (20) induced by an acceleration $|\mathbf{g}|=9.81 \mathrm{~m} / \mathrm{sec}^{2}$ corresponds to

$$
\nu_{T}=27.0 \mathrm{~Hz} \frac{l}{\mathrm{~mm}},
$$

where $l$ is the total length of the superconducting region (the length of the integration path in (26) joining the two sides of the junction), in which the field $\boldsymbol{E}_{T}$ exists.

Consider now the $n$th loop of the $1 \mathrm{D}$ array containing the junctions labeled $n$ and $n+1$, respectively. From (25) it follows

$$
\hbar \frac{d}{d t}\left(\varphi_{n}-\varphi_{n+1}\right)=-\oint_{\mathcal{C}_{n}}\langle\mathcal{E}, d \boldsymbol{r}\rangle
$$

where the path $\mathcal{C}_{n}$ circulates around the boundary of the surface element $\mathbf{S}_{n}$ just once. The electromotive force field $\mathcal{E}$ determines via (28) the temporal evolution of the difference of the variables $\varphi_{n}$ and $\varphi_{n+1}$ associated to the Josephson junctions in the considered loop. These $N-1$ equations describe the effects of the electromotive force field $\mathcal{E}$ (cf. (9)) on accelerated 1D parallel arrays of Josephson junctions.

The basic formula describing the effects of magnetomotive effects on superconducting interferometers is the condition of flux quantization. The generalized magnetic flux $\Phi_{n}$ through the area of the $n$th elementary loop $\mathbf{S}_{n}$ in an $1 \mathrm{D}$ parallel array determines via

$$
\hbar\left(\varphi_{n}-\varphi_{n+1}\right)=\Phi_{n}=\int_{S_{n}}\langle\mathcal{B}, d \mathbf{S}\rangle,
$$

the difference of the phase differences of the two junctions which form this loop. Taken severely this relation holds provided the superconducting loop is made of a material thick compared to the magnetic penetration depth $\lambda$. In this case there exists a path inside the wire connecting the junctions $n$ and $n+1$, on which the superfluid velocity field $\boldsymbol{v}_{s}$ becomes negligibly small. So, $\hbar \nabla \theta=\mathcal{A}$ along this path. In (29) $\Phi_{n}$ is the generalized flux from (18), incorporating both the conventional magnetic flux and (twice) the flux of the rotation field. Therefore superconducting interferometers can in principle be used to determine the rate of rotation $\Omega$ via the detection of the London spontaneous field $\mathbf{B}_{L}$ corresponding to $\Omega$ (cf. (13)).

By the generalized Faraday's law (10) the electromotive force field $\mathcal{E}$ along the integration path $\mathcal{C}_{n}$ that circulates the $n$th closed loop in the array just once is directly connected to the time derivative of the flux threading this area element

$$
\frac{d}{d t} \int_{S_{n}}\langle\mathcal{B}, d \boldsymbol{S}\rangle=-\oint_{\mathcal{C}_{n}}\langle\mathcal{E}, d \boldsymbol{r}\rangle
$$

A comparison of the time derivative of (29) with (28) indicates that these basic relations describing the effects of electromagnetomotive force fields on 1D parallel arrays are consistent with the generalized Faraday's law.

Using the RCSJ model (24) and Kirchhoff's rule, the total current $I_{b}$ flowing through the array is obtained as the phase sensitive superposition of the individual junction currents $I_{n}\left(\varphi_{n}\right)$

$$
I_{b}=\sum_{n=1}^{N} I_{n}\left(\varphi_{n}\right) .
$$

The gauge invariant phase differences $\varphi_{n}$ of adjacent Josephson junctions in the array are not independent, but are connected to each other by the condition of flux quantization (29). Neglecting the Biot-Savart type inductive couplings 17 among the currents flowing in the array, it follows from (29) that one can eliminate all phase variables $\varphi_{n}(t)$ in favor of a single phase variable, say 
$\phi(t)=\varphi_{1}(t)$. In this case (31) can be used to map the problem of $N$ coupled Josephson junctions onto a virtual single Josephson junction model and there results a scalar (RCSJ-like) differential equation determining the phase difference $\phi(t)^{17}$.

The decisive quantity determining the response of the 1D parallel Josephson junction array on magnetomotive force fields (22) is the complex structure factor $\mathcal{S}_{N}(\mathcal{B})^{17}$, given by

$$
\mathcal{S}_{N}(\mathcal{B})=\frac{1}{N} \sum_{n=1}^{N} \frac{I_{c, n}}{I_{c}} \exp \left[\frac{i}{\hbar} \sum_{m=0}^{n-1}\left\langle\mathcal{B}, \boldsymbol{S}_{m}\right\rangle\right],
$$

where the critical currents of the individual junctions are $I_{c, n}$ (their average over all $N$ junctions is $I_{c}$ ) and $\mathbf{S}_{m}$ are the orientated area elements of the array (with $\mathbf{S}_{0}=0$ ). The quantity $\mathcal{S}_{N}$ is strongly affected by the geometry of the array, i.e. the choice of the individual area elements $\mathbf{S}_{m}$ (cf. Fig. 2), and describes interference effects between the array junction currents in the presence of magnetomotive force fields.

In the overdamped junction regime and for static magnetomotive fields the scalar differential equation of the single (virtual) Josephson junction model can be solved analytically under conditions where a constant current $I_{b}$ is biased. The solution $\phi(t)$ then determines via $V(t)=\hbar / 2|e| \partial_{t} \phi(t)$ the voltage drop between the electrodes of the array. It turns out $\frac{17}{}$, that if the bias current $I_{b}$ exceeds the maximal critical array current $N I_{c}\left|\mathcal{S}_{N}(\mathcal{B})\right|$, the absolute value $\left|\mathcal{S}_{N}(\mathcal{B})\right|$ influences the time averaged voltage $\langle V\rangle$ across the array by

$$
\frac{h \nu}{2|e|}=\langle V\rangle=I_{c} R \sqrt{\left(\frac{I_{b}}{N I_{c}}\right)^{2}-\left|\mathcal{S}_{N}(\mathcal{B})\right|^{2}} .
$$

Here $\mathrm{R}$ denotes the average ohmic resistance of all array junctions. Taking into account all inductive couplings, the qualitative behavior of the array voltage response does get not affected, i.e., (33) also qualitatively describes the voltage response in this case ${ }^{17}$.

If the bias current $I_{b}$ is adjusted slightly above the array critical current, the presence of magnetomotive force fields gives an effect of shifting the frequency (respectively the voltage) which is orders of magnitude larger than the frequency shift displayed in (28). The relevant quantity here is the maximum voltage transfer factor of the voltage response function:

$$
\mathcal{T}_{N}=\left|\frac{\partial(2|e|\langle V\rangle)}{\partial \mathcal{B}}\right|_{\max }
$$

which determines the maximum sensitivity of the array on magnetomotive fields. Scaling (33) in experimentally relevant units, we have for $|\boldsymbol{\Omega}|=2 \pi / \mathrm{sec}$ that the magnetomotive field (14), i.e. the London spontaneous field $\boldsymbol{B}_{L}$, corresponds to

$$
\nu_{L}=3.46 \cdot 10^{4} \mathrm{~Hz} \frac{\mathcal{T}_{N}}{\text { Volt } / \text { Tesla }}
$$

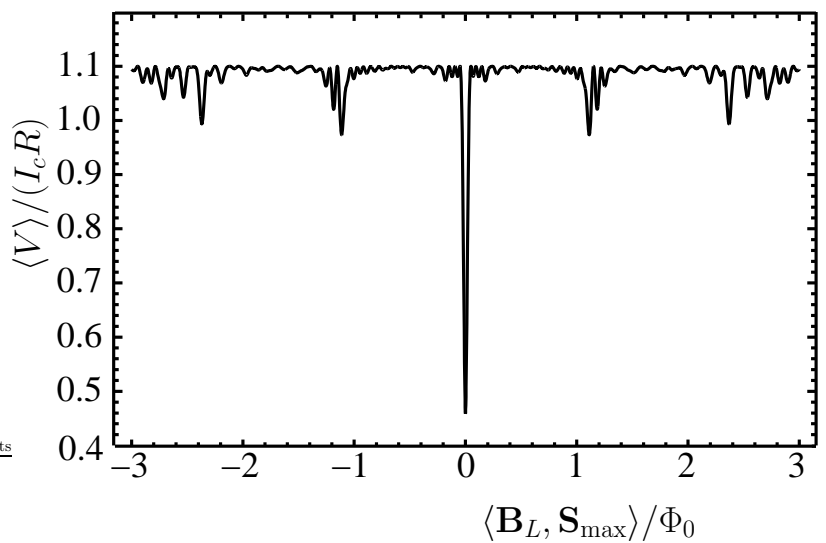

FIG. 3: Voltage response due to rotation of a quantum interference filter, with $N=30$ (overdamped) Josephson junctions for bias current $I_{b}=1.1 N I_{c}$. The time averaged voltage $\langle V\rangle$ in units of $I_{c} R$ is plotted versus the normalized magnetic flux $\left\langle\mathbf{B}_{L}, \mathbf{S}_{\max }\right\rangle / \Phi_{0}$ which the London spontaneous field $\mathbf{B}_{L}$ induces in the largest area element $\mathbf{S}_{\max }$ of the array. The loop areas $\left|\mathbf{S}_{n}\right|$ are all in plane and randomly distributed between 0.1 and $1.0\left|\mathbf{S}_{\max }\right|$.

provided the array is driven at its most sensitive point of operation. Typical experimental values for the transfer factor of bare 1D parallel arrays (with $N=30$ ) are of the order of $\mathcal{T}_{N} \approx 10^{2}-10^{3}$ Volt/Tesla 18 . As can be derived from (33) the transfer factor scales with the number $N$ of junctions in the array, so that $\mathcal{T}_{N}$ can be increased with $N$. Using additional flux-focussing structures, e.g. superconducting pick-up loops, the transfer factor can be further increased by several orders of magnitudes up to $\mathcal{T}_{N} \approx 10^{6}$ Volt/Tesla. According to (35), such devices are then very sensitive to rotations and can measure the angular velocity $\boldsymbol{\Omega}$ very precisely.

In Fig. 3 the voltage response, according to (33), of a quantum interference filter due to rotation with angular velocity $\boldsymbol{\Omega}$ is shown. For vanishing magnetic field $\mathbf{B}=0$, the normalized voltage $\langle V\rangle /\left(I_{c} R\right)$ is plotted versus the normalized magnetic flux $\left\langle\mathbf{B}_{L}, \mathbf{S}_{\max }\right\rangle / \Phi_{0}$ the London spontaneous field $\mathbf{B}_{L}=-2 m / q \boldsymbol{\Omega}$ induces in the largest area element $\mathbf{S}_{\max }$ of the array. The SQIF contains $N=30$ junctions and the loop areas $S_{n}=\left|\mathbf{S}_{n}\right|$ are randomly distributed between 0.1 and $1.0\left|\mathbf{S}_{\max }\right|$. For maximal voltage swing the bias current $I_{b}$ is adjusted slightly above $N I_{c}$.

For vanishing magnetic field $\mathbf{B}=0$, the voltage response is indeed a unique function of the London field $\left|\mathbf{B}_{L}\right|$ and hence of $|\boldsymbol{\Omega}|$ around its global minimum at $\left|\mathbf{B}_{L}\right|=0$. This suggests that it would be possible, e.g. by measuring control current(s) flowing through the wires of suitably orientated compensation coil(s), to reconstruct the absolute value, the orientation and even the phase of the rate of rotation, i.e. to determine the full angular velocity vector $\boldsymbol{\Omega}(t)$ and its time dependence.

A basic problem for the sensitive detection of rotation and other noninertial fields is that the device has to be 
shielded against external magnetic fields, like that of the earth. It has been shown in Ref ${ }^{24}$ by Satterthwaite and Gawlinski for the stationary case, that superconducting shielding, which delivers the shielding factors required to detect rotations as slow as, e.g., that of the earth, implies that the apparatus cannot distinguish between applied rotation and applied proper magnetic flux: The co-rotating superconducting shield prevents such a distinction. The current induced by a rotation with $\boldsymbol{\Omega}$ is the same as that induced by an external, applied magnetic field $-\boldsymbol{B}_{L}$, because the device cannot tell from which of the two parts of $\mathcal{B}$ the induced current actually comes from. Shielding is not necessary (and, indeed, not possible) for charge neutral quantum interference devices ${ }^{25}$, which are thus capable of detecting absolute rotation, whereas superconductively shielded SQUIDs or Josephson arrays are not. What is measurable by superconducting interferometers are the fields $\mathcal{B}$ and $\mathcal{E}$ coming from the (accelerated) motion relative to the external shield, which remains fixed with respect to the local frame of inertia. In particular, SQIFs can measure relative motion on an absolute scale. However, a gyromagnetic gyroscope based on this idea needs some mechanics and therefore can not be more sensitive than a mechanical gyroscope itself.

One possibility to build a gyromagnetic gyroscope without any moving parts, is to use a material with a magnetomechanic factor $\gamma$ different from the factor $\gamma=-2 m / q$, occurring in the London equation (13) for the superconducting shield. In this case it is possible to circumvent the problem of indistinguishability, i.e. to which parts of $\mathcal{B}$ an induced current is related. For example a ferromagnetic material, whose magnetic field induced by rotation is itself measured by a SQUID which is shielded by a superconducting shield, can measure the rotation field ${ }^{26}$. But such a device is not a superconducting gyroscope in a narrower sense ${ }^{24}$ which exclusively relies on the superconductors' response to rotation.

\section{TRAJECTORY TRACKING}

The determination of the trajectory $x^{i}(t)+x_{0}^{i}(t)$ of an electron (or Cooper pair) in an accelerated superconductor amounts to solving the second order differential equation

$$
\frac{d^{2} x^{i}}{d t^{2}}+\frac{q}{m} \epsilon_{i j k}\left(\boldsymbol{B}_{L}\right)_{k} \frac{d x^{j}}{d t}+\frac{q}{m}\left(\boldsymbol{E}_{T}\right)_{i}=0
$$

which is (11) with $\boldsymbol{F}=m d^{2} x^{i} / d t^{2}$, where the in general time dependent proper magnetic and electric fields $\boldsymbol{B}_{L}$ and $\boldsymbol{E}_{T}$ are determined from Eqs. (13) and (19). The electric field $\boldsymbol{E}_{T}$ is, according to (19), in the rotational part position dependent, linear in the distance vector $\boldsymbol{r}=x^{i} \boldsymbol{e}_{i}$ from the center of (local) solid body rotation 27 . In the appendix, equation (36) is explained in terms the geodesic equation (37), and the fields $\boldsymbol{E}_{T}$ and
$\boldsymbol{B}_{L}$ are identified as connection coefficients on a Riemannian manifold, i.e., on a manifold representing space-time with some metric coefficients.

\section{CONCLUSIONS}

Moving superconducting circuits consisting of current biased superconducting quantum interference filters (SQIFs) are local oscillators that undergo a characteristic and unique change of their oscillation frequency under acceleration and rotation. However, by the very nature of the combined vector field $\mathcal{A}$, Eq.(6), such a superconducting interferometer is only capable to detect the combined magnetomotive field $\mathcal{B}$, Eq.(22), and the combined electromotive field $\mathcal{E}$, Eq.(21). Employing a suitable shield such SQIFs can nevertheless measure relative motion on an absolute scale. The aforementioned devices might be used, for example, to construct an absolute detector of noninertial motion in the context of seismology.

\section{NONINERTIAL FORCE FIELDS FROM THE GEODESIC EQUATION}

The motion of a test particle, upon which no external (electromagnetic) force is acting, is describable by the geodesic equation in space-time,

$$
\frac{d u^{\mu}}{d \tau}+\Gamma_{\alpha \beta}^{\mu} u^{\alpha} u^{\beta}=0
$$

with the four-velocity normalized to unity, i.e. $u^{\mu} u_{\mu}=$ -1 . The connection coefficients $\Gamma^{\mu}{ }_{\alpha \beta}$ serve to describe any kind of "acceleration" $d u^{\alpha} / d \tau$, caused by the transformation to the curvilinear co-ordinates of a rotating and accelerating frame (cf. equation (11) valid for a rigidly rotating frame), and nonrelativistic particle velocities. The true, invariant four-acceleration is invariantly zero for a geodesic: The equation above describes the (kinematic) autoparallel property of the four-velocity with components $u^{\alpha}$. If electromagnetic fields are present, the right hand side of (37) is no longer zero, and the covariant Lorentz force four-acceleration equation on a particle of charge $q$ and inertial rest mass $m$ in the presence of an electromagnetic field acting on the particle reads

$$
m \frac{d u^{\mu}}{d \tau}=\left(q F_{\nu}^{\mu}-m \Gamma_{\alpha \nu}^{\mu} u^{\alpha}\right) u^{\nu}
$$

where we have brought the connection coefficient term to the right hand side.

In the weak field limit $g_{\mu \nu} \simeq \eta_{\mu \nu}+h_{\mu \nu}$, in which raising and lowering of indices is to lowest order in $\left|h_{\mu \nu}\right| \ll\left|\eta_{\mu \nu}\right|$ accomplished by $\eta_{\mu \nu}=\operatorname{diag}(-1,1,1,1)$, the connection coefficients take on the form

$$
\Gamma_{\alpha \nu}^{\mu}=\frac{1}{2} \eta^{\mu \beta}\left(h_{\beta \alpha, \nu}+h_{\beta \nu, \alpha}-h_{\alpha \nu, \beta}\right) .
$$


We now use that the spatial components of (37) $\operatorname{are}^{28}$

$$
\begin{aligned}
\frac{d^{2} x^{i}}{d t^{2}}= & -\Gamma_{00}^{i}-2 \Gamma_{0 j}^{i} \frac{d x^{j}}{d t}-\Gamma^{i}{ }_{j k} \frac{d x^{j}}{d t} \frac{d x^{k}}{d t} \\
& +\left[\Gamma_{00}^{0}+2 \Gamma^{0}{ }_{0 j} \frac{d x^{j}}{d t}+\Gamma^{0}{ }_{j k} \frac{d x^{j}}{d t} \frac{d x^{k}}{d t}\right] \frac{d x^{i}}{d t} \\
\simeq & -\Gamma_{00}^{i}-2 \Gamma_{0 j}^{i} \frac{d x^{j}}{d t}
\end{aligned}
$$

The last line holds if we consider the lowest (linear) order in the charge velocity $\boldsymbol{v}$, whose magnitude is assumed to be much less than the speed of light. The rotation rate $\boldsymbol{\Omega}$, i.e., the invariant (vorticity) measure of the proper velocity $\boldsymbol{\Omega} \times \boldsymbol{r}$ induced by rotation, is taken into account up to $O\left(\mathbf{\Omega}^{2}\right)$, in the form of $\Gamma_{00}^{i}$. Terms which are quadratic in $\boldsymbol{v}$ (last term in the first line of (40)), and those of higher order than quadratic in $\boldsymbol{\Omega}$ and $\boldsymbol{v}$ and their products (terms in the second line of (40)) are neglected.

Equation (38) then gives the following spatial components

$$
\begin{gathered}
m \frac{d^{2} x^{k}}{d t^{2}}=q F_{k 0}-m \Gamma_{00}^{k}+\left(q F_{k i}-2 m \Gamma_{0 i}^{k}\right) v^{i} \\
=q\left(F_{k 0}+F_{k i} v^{i}\right)-m\left(h_{k 0,0}-\frac{1}{2} h_{00, k}\right) \\
-m\left(h_{k 0, i}+h_{k i, 0}-h_{0 i, k}\right) v^{i}
\end{gathered}
$$

This relation leads to the generalized electromotive and magnetomotive force fields, reinstating the speed of light $c$, and neglecting the time derivative of $h_{i k}$,

$$
\begin{aligned}
\mathcal{E} & =q \boldsymbol{E}+\frac{1}{2} m c^{2} \nabla h_{00}-m c \partial_{t} \boldsymbol{h}_{0}, \\
\boldsymbol{v} \times \mathcal{B} & =\boldsymbol{v} \times\left(q \boldsymbol{B}+m c \nabla \times \boldsymbol{h}_{0}\right) .
\end{aligned}
$$

The relations (43) result in the following identifications with the potentials in (5):

$$
\frac{1}{2} c^{2} h_{00}=a_{0}, \quad c \boldsymbol{h}_{0}=\boldsymbol{a} .
$$

Measuring the fields $\boldsymbol{B}_{L}$ and $\boldsymbol{E}_{T}$ in (13) and (19) thus yields the connection coefficients $\Gamma_{00}^{i}$ and $\Gamma_{0 j}^{i}$ (spatial coordinates are in a Cartesian frame):

$$
\begin{aligned}
\left(\boldsymbol{E}_{T}\right)_{k} & =\frac{m}{q} \Gamma_{00}^{k} \\
\left(\boldsymbol{B}_{L}\right)_{j} \epsilon_{i k j} & =-2 \frac{m}{q}(\boldsymbol{\Omega})_{j} \epsilon_{i k j}=-\frac{2 m}{q} \Gamma_{0 i}^{k}
\end{aligned}
$$

For the distinction and understanding of "real" electromagnetism and generalized electromagnetism as expounded here, it is of importance to bear in mind that $\boldsymbol{E}_{T}$ and $\boldsymbol{B}_{L}$, if understood as connection coefficients like in the relations above, have no exact co-ordinate invariant meaning as tensor fields like the proper electromagnetic fields $\boldsymbol{E}$ and $\boldsymbol{B}$ have. They gain an approximate co-ordinate invariant meaning only in the weak field limit $\left|h_{\mu \nu}\right| \ll\left|\eta_{\mu \nu}\right|$, and if $\partial_{t} h_{i k}$ is negligible, because in this limit the $\Gamma_{\beta \gamma}^{\alpha}$ in (39) transform tensorially, and the field strength is identified to be

$$
\begin{aligned}
-\Gamma_{\mu 0 \nu} & =\Gamma_{\nu 0 \mu}=-\frac{1}{2}\left(h_{0 \mu, \nu}+h_{\mu \nu, 0}-h_{0 \nu, \mu}\right) \\
& =\mathcal{F}_{\mu \nu}=\partial_{\mu} \mathcal{A}_{\nu}-\partial_{\nu} \mathcal{A}_{\mu} .
\end{aligned}
$$

In the specified limit of small velocities and small deviations from Minkowski space-time, we can thus ascribe co-ordinate invariant, i.e. tensorial meaning to $\mathcal{F}_{\mu \nu}$.
1 J. C. Maxwell: A Treatise on Electricity and Magnetism (Dover, New York, 1954, originally published 1891).

2 S. J. Barnett: Magnetization by rotation, Phys. Rev. 6, 239-270 (1915).

3 R. C. Tolman, T. D. Stewart: The electromotive force produced by the acceleration of metals, Phys. Rev. 8, 97-116 (1916).

4 R. C. Tolman, T. D. Stewart: Mass of electric carrier in copper, silver, aluminum, Phys. Rev. 9, 164-167 (1917).

${ }^{5}$ G. F. Moorhead, G. I. Opat: Electric fields in accelerating conductors: measurement of the EMF in rotationally accelerating coils, Class. Quantum Grav. 13, 3129-3139 (1996).

6 An overview of the existing literature (up to the year 1992) may be found in T. W. Darling, F. Rossi, G. I. Opat, G. F. Moorhead: The fall of charged particles under gravity: A study of experimental problems, Rev. Mod. Phys. 64, 237-257 (1992).

7 F. London: Superfluids, Volume 1: Macroscopic Theory of Superconductivity (2nd edition, Dover, New York, 1961).

8 J. E. Zimmerman, J. E. Mercereau: Compton Wavelength of Superconducting Electrons, Phys. Rev. Lett. 14, 887-888 (1965).
9 M. Liu: Rotating Superconductors and the FrameIndependent London Equation, Phys. Rev. Lett. 81, 32233226 (1998).

10 J. Tate, S. B. Felch, B. Cabrera: Determination of the Cooper-pair mass in niobium, Phys. Rev. B 42, 7885-7893 (1990).

11 A. A. Verheijen, J. M. van Ruitenbeek, R. de Bruyn Ouboter, L. J. de Jongh: Measurement of the London moment in two high-temperature superconductors, Nature 345, 418-419 (1990).

12 M. A. Sanzari, H. L. Cui, F. Karwacki: London moment for heavy-fermion superconductors, Appl. Phys. Lett. 68, 3802-3804 (1996).

13 R. B. Laughlin, D. Pines: The Theory of Everything, Proc. Natl. Acad. Sci. USA 97, 28-31 (2000).

14 A. Hartland et al.: Direct Comparison of the Quantized Hall Resistance in Gallium Arsenide and Silicon, Phys. Rev. Lett. 66, 969-973 (1991).

15 A. K. Jain, J. E. Lukens, J.-S. Tsai: Test for Relativistic Gravitational Effects on Charged Particles, Phys. Rev. Lett. 58, 1165 (1987).

16 U. R. Fischer, N. Schopohl: Hall state quantization in a 
rotating frame, Europhys. Lett. 54, 502 (2001).

17 J. Oppenländer, Ch. Häussler, N. Schopohl: Non$\Phi_{0}$-periodic macroscopic quantum interference in onedimensional parallel Josephson junction arrays with unconventional grating structure, Phys. Rev. B 63, 024511 (2001).

18 J. Oppenländer, T. Träuble, Ch. Häussler, N. Schopohl: Superconducting Multiple Loop Quantum Interferometers, IEEE Trans. Appl. Supercond. 11, 1271-1274 (2001); Ch. Häussler, T. Träuble, J. Oppenländer, N. Schopohl: $L C$ Resonant Voltage Response of Superconducting Quantum Interference Filters, IEEE Trans. Appl. Supercond. 11, 1275-1278 (2001).

19 Ch. Häussler, J. Oppenländer, and N. Schopohl: Nonperiodic flux to voltage conversion of series arrays of dc superconducting quantum interference devices, J. Appl. Phys. 89, 1875-1879 (2001).

${ }^{20}$ We omit a factor of $1 / 2$ in the definition of the vector potential which can be introduced for (weak) gravity as a tensorial, spin-2 theory, cf., e.g., B. Mashhoon: Gravitoelectromagnetism, gr-qc/0011014 in Reference Frames and Gravitomagnetism, edited by J.-F. Pascua-Sánchez, L. Floría, A. San Miguel, and F. Vicente (World Scientific, Singapore, 2001), pp. 121-132, and references cited therein, as well as the Appendix of the present paper.

21 E. J. Post: Sagnac Effect, Rev. Mod. Phys. 39, 475-493 (1967).
22 G. E. Stedman: Ring-laser tests of fundamental physics and geophysics, Rep. Prog. Phys. 60, 615-688 (1997).

23 K.K. Likharev: Dynamics of Josephson Junctions and Circuits (Gordon and Breach Science Publishers, New York, 2nd printing, 1991).

24 J. C. Satterthwaite, E. T. Gawlinski: Concerning superconducting inertial guidance gyroscopes inside superconducting magnetic shields, J. Appl. Phys. 82, 5829-5836 (1997).

25 O. Avenel, E. Varoquaux: Detection of the Earth Rotation with a Superfluid Double-hole Resonator, Czech. J. Phys. 46 Suppl. S6, 3319-3320 (1996); K. Schwab, N. Bruckner, R. E. Packard: Detection of the Earth's rotation using superfluid phase coherence, Nature 386, 585 (1997); Y. Mukharsky, O. Avenel, É. Varoquaux: Rotation measurements with a superfluid ${ }^{3}$ He gyrometer, Physica B 284288, 287-288 (2000); R. W. Simmonds, A. Marchenkov, E. Hoskinson, J. C. Davis, R. E. Packard: Quantum interference of superfluid ${ }^{3} \mathrm{He}$, Nature 412, 55-58 (2001).

26 S. Vitale, M. Bonaldi, P. Falferi, G. A. Prodi, M. Cerdonio: Magnetization by rotation and gyromagnetic gyroscopes, Phys. Rev. B 39, 11993-12002 (1989).

27 This dependence lifts the degeneracy of the Landau energy levels related to $\mathcal{B}$.

28 S. Weinberg: Gravitation and Cosmology (Wiley and Sons, New York, 1972), §9.1. 